\title{
EPIC DIMENSIONS: A COMPARATIVE ANALYSIS OF 3D ACQUISITION METHODS
}

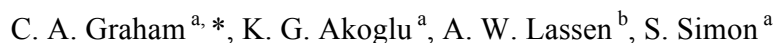

${ }^{a}$ Institute for the Preservation of Cultural Heritage, Yale University, USA - (c.graham, goze.akoglu, stefan.simon)@yale.edu

${ }^{\mathrm{b}}$ Yale Babylonian Collection, Yale University, USA - agnete.lassen@yale.edu

KEY WORDS: Structured Light Scanning, Triangulation Laser Scanning, Photometric Stereo, Close-range Photogrammetry, Deviation Analysis, Geometric Accuracy, Dissemination, 3D Printing

\begin{abstract}
:
When it comes to capturing the geometry of a cultural heritage artifact, there is certainly no dearth of possible acquisition techniques. As technology has rapidly developed, the availability of intuitive 3D generating tools has increased exponentially and made it possible even for non-specialists to create many models quickly. Though the by-products of these different acquisition methods may be incongruent in terms of quality, these discrepancies are not problematic, as there are many applications of 3D models, each with their own set of requirements. Comparisons of high-resolution 3D models of an iconic Babylonian tablet, captured via four different closerange technologies discussed in this paper assess which methods of 3D digitization best suit specific intended purposes related to research, conservation and education. Taking into consideration repeatability, time and resource implications, qualitative and quantitative potential and ease of use, this paper presents a study of the strengths and weakness of structured light scanning, triangulation laser scanning, photometric stereo and close-range photogrammetry, in the context of interactive investigation, conditions monitoring, engagement, and dissemination.
\end{abstract}

\section{INTRODUCTION}

Virtualization presents innovative opportunities for enhanced investigation of and interaction with cultural heritage. Through 3D digitization, new possibilities for educational engagement, collaborative discovery and conditions monitoring have been introduced with implications for improved understanding and care of material culture. Due to the interactive nature of visualization techniques, including adoption into immersive virtual reality environments, integration into augmented reality applications and incorporation in web-hosted viewers, three dimensional (3D) models have steadily gained popularity. Parallel to this rise has been the proliferation of varied opensource, accessible technologies and software which enable the creation of 3D data.

With inspiration from other comparisons of digitization techniques and deliverables (Fassi et al., 2013; Menna et al., 2016), this paper presents the material considerations, logistical planning, acquisition campaigns, post-processing pipelines and applications of 3D assets yielded from four different close-range digitization methods and culminates in qualitative and quantitative analyses of their competences in a range of applications.

\subsection{Digitization Subject}

Offering a unique view into Ancient Mesopotamia and a pertinent example of material culture at risk, one of the world's earliest enduring literary works serves as the subject of this case study. This clay tablet from the Epic of Gilgamesh, YBC 2178, is held in the Yale Babylonian Collection and dates to around 1800 BCE (Fig. 1). The tablet measures $22.6 \times 17.0 \times 3.7$ millimeters $(\mathrm{mm})$ and is missing sections in the middle and toward the bottom due to breakage. The dimensions and condition of the tablet present occlusions that can be difficult to capture via some acquisition methods.

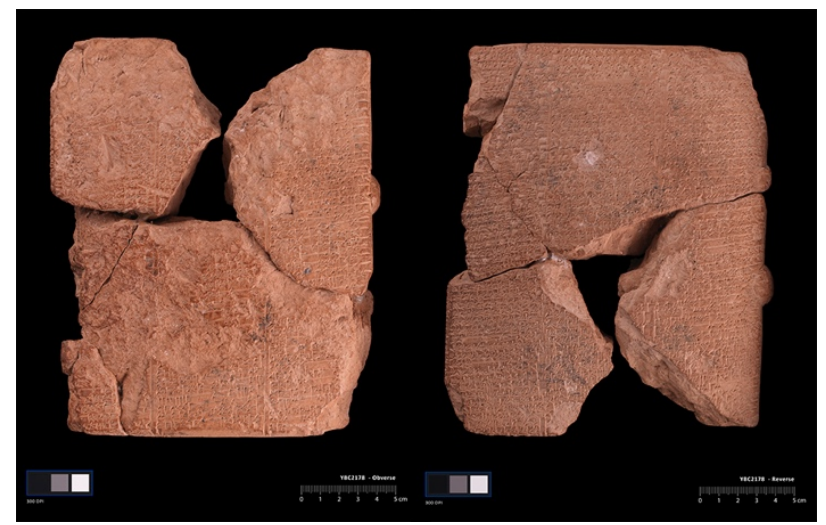

Figure 1. High-resolution photographs of the obverse and reverse of YBC 2178. (credit: Yale Babylonian Collection)

Although, for the most part, the tablet retains its material integrity, in the past it has incurred damage and been restored on many occasions. Conservation treatments and consolidations have rendered visible cracks and areas of perceptible adhesive, which pose geometric complexities and specular challenges for $3 \mathrm{D}$ acquisition technologies.

\subsection{Project Approaches and Aims}

Scanning, photography and reflectance transformation imaging were undertaken in order to capture scaled geometric and textural data in optically distinct ways. The manner in which light, laser and projected pattern interacted with the texture of the clay tablet yielded differences in the raw data acquired, and therefore the quality and fidelity of the post processed final models. Quantitative and qualitative comparisons were undertaken to discern deviation between meshes, which helped to inform assessment of their effectiveness in a variety of applications.

\footnotetext{
* Corresponding author
} 
These applications are as diverse as their requirements. For scholars of lost languages enhanced legibility, which relies upon a robust texture map, is significant. On the other hand, researchers who pursue comparative analyses require a highly precise geometric $3 \mathrm{D}$ representation in order to make their calculations. Conservators prioritize reconstruction and conditions monitoring and therefore value a standard, repeatable pipeline which yields consistent high-resolution results.

At this time, the detail which can be acquired by many digitization methods far exceeds that which can be accommodated by most 3D printers and web-hosted viewers. Lower resolution models are sufficient for 3D printing and web based platforms, which is mainly of interest to educators and curators who wish to employ tactile 3D prints for their didactic purposes. Embedded 3D models can be texture mapped with higher resolution color information to foster a greater sense of authenticity and engagement.

Although high resolution 3D models are desired for almost all applications, lower resolution models are also necessary for dissemination which make important discoveries, guided practices, and collaboration across the distances possible.

Our study demonstrates how an acquisition technique may be chosen depending factors including resource availability and the final use of the 3D model.

\section{DATA ACQUISITION: PLANNING, EXECUTION, AND POST-PROCESSING}

Taking into consideration the small size, detailed geometry and varied texture of YBC 2178 and its associated complexities along with the desire to acquire high resolution data at a scale of one to one, acquisition campaigns via structured light scanning, triangulation laser scanning, photometric stereo and close-range photogrammetry were undertaken.

\subsection{Structured Light Scanning}

Based on the complex structure, small size and texture of YBC 2178, a handheld structured light scanner was thought to be a powerful instrument for capturing geometric and textual detail in high-resolution. The Artec Space Spider was chosen due to its internal temperature stabilization feature, which yields high accuracy and long-term repeatability. With a working distance of 0.2-0.3 meters (m) and an optical resolution of up to $0.1 \mathrm{~mm}$, this scanner specializes in the digitization of small geometric details. The scanner operates with a blue light-emitting diode (LED) light source at a wavelength 460 nano meters $(\mathrm{nm})$ and is capable of capturing up to 7.5 frames per second (fps). This technology enables texture to be captured at a resolution of 1.3 mega pixels and a color depth of 24 bits per pixel (bpp).

Scanning took place without any additional specialized lighting at the Yale Babylonian Collection over the course of two hours in 2017. The tracking of geometry and texture was selected as data was collected for each side separately. The tablet was slowly rotated $360^{\circ}$ while the acclimatized scanner was held a consistent distance from the object and moved in a pattern in order to capture the difficult central geometry resultant from past breaks.

The scans were then post-processed manually in Artec Studio 12 Professional, where cleaning, alignment, sharp fusion with a resolution of $0.1 \mathrm{~mm}$, and texture mapping were carried out to yield a final high-resolution, detailed 3D model. The exported model was then decimated in MeshLab 2016.12 (Cignoni et al., 2008) via poisson disk sampling (Corsini et al., 2012) and screened poisson surface reconstruction (Kazdhan et al., 2013) to yield three textured, downsampled versions for web viewing and $3 \mathrm{D}$ printing.

\subsection{Triangulation Laser Scanning}

Due to intricate morphology of YBC 2178, our highest resolution triangulation laser scanner was employed for capture of geometry. The ShapeGrabber SG1002 Scanhead captures at an optical resolution of about $0.025 \mathrm{~mm}$ in a field of view of 0.2$0.64 \mathrm{~m}$. The wavelength range for the SG1002 laser diode is 650 $670 \mathrm{~nm}$.

In 2015, an approximately 1.5 hour acquisition campaign took place and was repeated on a second occasion due to initial quality concerns. The triangulation laser scanning acquisitions were carried out in a light-controlled project room of the Institute for the Preservation of Cultural Heritage's (IPCH) Digitization Lab. Since this scanner does not acquire texture data, lower lighting was selected. Individual range maps were captured with approximately $20^{\circ}$ overlap $360^{\circ}$ around the object from three different angles. Twenty-four scans were acquired in total and exported into a non-proprietary file type for post-processing.

Post-processing was carried out entirely in MeshLab 1.3.3. Here scans were registered via the Iterative Closest Point (ICP) alignment algorithm (Besl and McKay, 1992; Zhang, 1994), cleaned, merged, refined, and meshed via poisson surface reconstruction. Once the most detailed model possible was generated, color information from high-resolution photographs was texture-mapped onto the model (Ranzuglia et al., 2013). The raster images were seamlessly projected to the geometry with the exception of the occluded areas which had eluded photography. These areas correspond to the broken interior of the tablet and remain white on the final 3D model. The resultant 3D model did not require downsampling for $3 \mathrm{D}$ printing, however a slight decimation was required for upload to a web based viewer.

\subsection{Photometric Stereo}

Given the low relief geometry of the inscriptions on the tablet, Reflectance Transformation Imaging (RTI) of the tablet was captured in the context of another project in 2015. Raw data was acquired under an RTI dome equipped with a 45 light array. Forty-five images were captured per side with Canon EOS 5D Mark III DSLR at a focal length of $70 \mathrm{~mm}$.

The raw data from this acquisition was then processed via RTI23D. The core library behind this software was developed at the Center for Advanced Studies, Research and Development in Sardinia (CRS4) based on a MATLAB code written by Pintus et al. (2006) that applies photometric stereo to data derived from scanning electron microscope. A later developed graphical user interface enables streamlined processing of scaled 3D models given a mask and measurement factor. Two 3D models, one corresponding to the obverse and on to the reverse side of the tablet, were generated with this approach. It is not possible to combine the two sides into one comprehensive 3D model due to both a lack and distortion of data. Coupled with time spent on acquisition, approximately 1 hour was spent in the development of these extremely detailed 3D models. The models necessitated downsampling and additional processing for dissemination purposes. 


\subsection{Close-range Photogrammetry}

While visually enlightening, a single photograph is in many ways anecdotal and can be biased by the perceptions of the photographer (Mudge et al., 2006). Photogrammetry is the art and science of obtaining precise mathematical measurements and 3D data from two or more photographs (ASP, 1966). Photogrammetric techniques can be applied to virtually any source of imagery. The basic requirement for photogrammetry is an overlapping pair of photographs. Photogrammetric techniques can be applied to any photography, free from their scale or vantage point. Resulting datasets can be viewed, manipulated, and measured with available open-source software.

The rapid evolution of digital cameras and increasing capabilities of computers and analytical software have dramatically expanded the variety of situations to which photogrammetry may be applied, while simultaneously decreasing the costs of acquisition, processing, and analysis. Within the last decade, advances in digital imagery and photogrammetric software, combined with lower equipment costs (Matthews et al., 2004), have enabled the use of photogrammetric methods over a wider range of applications, while at the same time reducing equipment costs and decreasing computing times, making the entire process more cost-effective.

In cultural heritage studies documenting and evaluating the present condition, and in some instances, past condition, is the main use of close-range photogrammetry (CRP). By definition, $\mathrm{CRP}$ is simply photogrammetric data collection and processing where the subject is less than $300 \mathrm{~m}$ away and the size of the object is relatively small compared to buildings and structures (Luhmann et al., 2006). This condition dataset provides information on the current state of an object as a basis for future decision making, moreover it also provides a tool for determining the effectiveness of conservation treatments. In that sense, the data from CRP is quantifiable and repeatableessential digitization criteria to the conservation related fields.

For YBC 2178, CRP was conducted to make the comparison and to reveal the efficiency of the technique for different uses of its 3D model. Photography was acquired using a Canon EOS 5D Mark III DSLR at a focal length of $100 \mathrm{~mm}$ in entirely manual mode of the camera in 2017. A total of 337 images were acquired for the full dataset, comprising high resolution .jpg files 180 of which were used for the model generation.

The photographs were processed using Agisoft Photoscan Professional V 1.2.0 on a work station with $3.20 \mathrm{GHz}$ processor and 16.0 GB RAM. The photographs were first masked before the alignment process to remove potentially misleading data for the matching algorithms. The data were divided into chunks, mainly one for each side of the tablet then the chunks were aligned and merged to one comprehensive model with the help of reference markers placed in each chunk (Fig. 2). The final model did not require additional processing for dissemination.

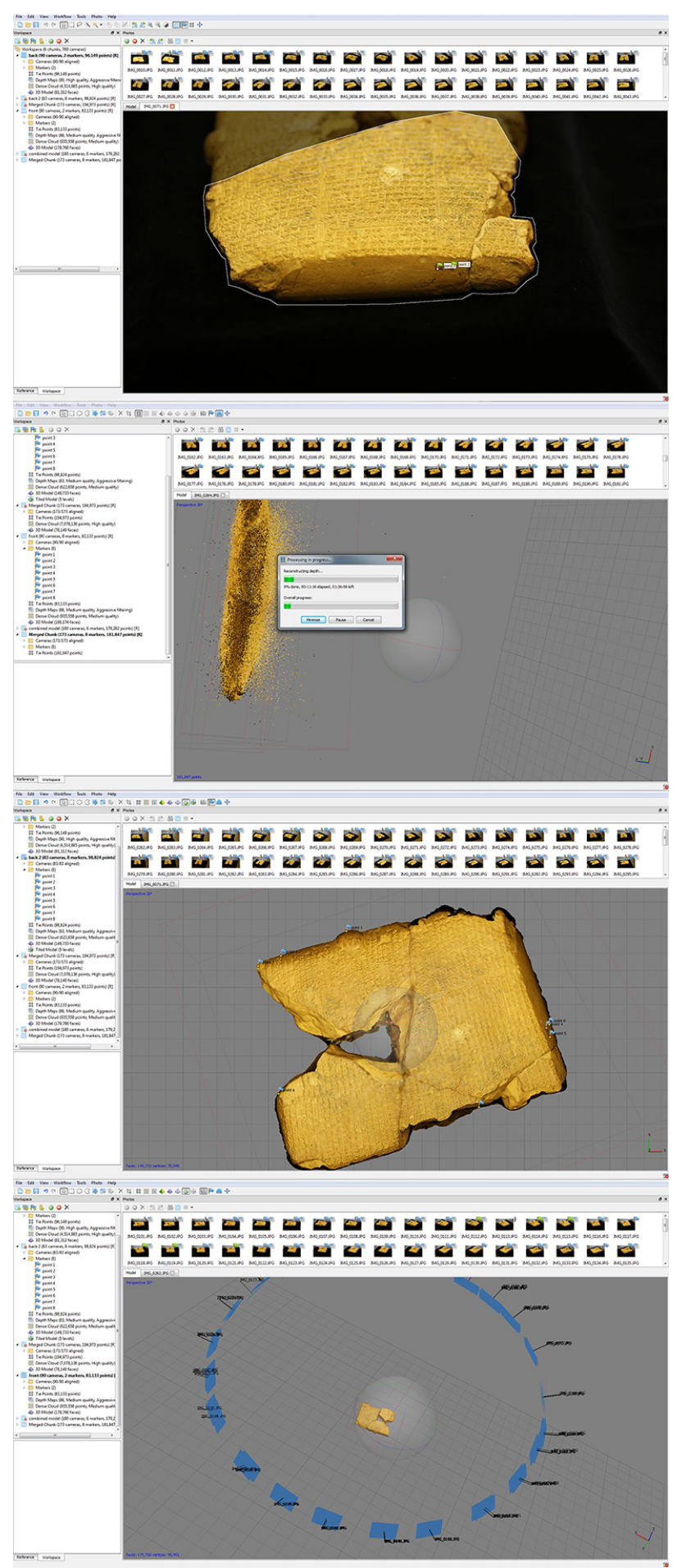

Figure 2. Screenshots from Agisoft Photoscan taken during processing. 


\section{COMPARISONS}

Qualitative and quantitative comparisons were undertaken to evaluate the accuracy and fidelity of geometry and texture for technical applications as well as the success of 3D printing and web-hosted visualization for more accessible applications.

\subsection{Geometry}

3.1.1 Geometric Accuracy: In order to assess the accuracy of the 3D data, models were opened in MeshLab and measurements of individual tablets were taken and compared to the documented dimensions of the tablet. For the 3D models generated via structured light scanning, triangulation laser scanning, and CRP the ratios of measurements taken correspond to those of the tablet, therefore they can be deemed quite accurate in size. Despite scaling in the RTI23D software, the 3D models yielded from photometric stereo were smaller and both needed to be scaled up $108 \%$ in order to match the length and width of the others.

3D models were then uploaded into a MeshLab project file and aligned to one another. Geometric differences between the meshes were computed according to Hausdorff distance measure and ascribed a color value (Cignoni et al., 1998). Figures 3 and 4 represent distance via color for a selection of these calculations. Blue signifies the areas of greatest similarity and red denotes areas where there is the greatest divergence between 3D models.

The 3D models generated via structured light and triangulation laser scanner (Fig. 3) are nearly identical in dimension and outline. Divergences arise due to the difference in resolution of the models. This can be seen in the presence of light blue and turquoise corresponding to inscriptions. The $3 \mathrm{D}$ model generated via CRP is also precise in dimension, however due to occlusions and difficulty imaging angles corresponding to the broken regions, there is extraneous detail in parts of the center. A similar trend corresponding to the lack of detail of the inscriptions can also be witnessed in the Hausdorff depictions between this model and that from the structured light. On this scale, the structured light 3D model has a highest geometric fidelity.

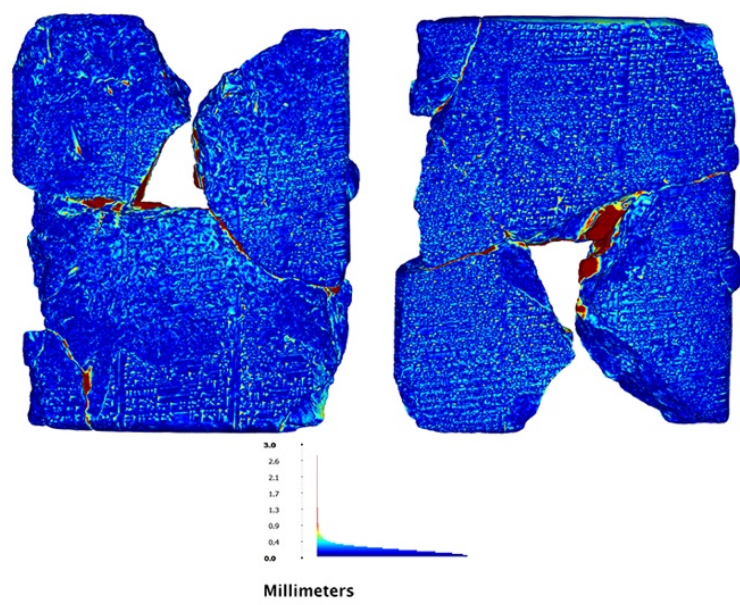

Figure 3. Color representations of Hausdorff distance calculated from the structured light 3D model to the triangulation laser scanner 3D model.

Even with the aforementioned increase in scale, the 3D data yielded via photometric stereo (Fig. 4) does not fall in line with that of the others. Due to the simplicity of assumptions behind the mathematical model used in RTI23D (a Lambertian materiality, a bas-relief surface property, a point light source at infinity, and an orthographic camera projection), this software does not yield quantitatively reliable results. Since it was created for other applications, global distortion is experienced and geometric accuracy is not achieved. The 3D models created via photometric stereo portray a trend toward flattening in the center and curving of the edges. This is evidenced by the concentration of increasing Hausdorff distance in the center and edges of both obverse and reverse sides of the tablet in Fig. 4. Nearly identical results in trend and measurement were derived from calculation of Hausdorff distances recorded between the two photometric stereo models and each of the other datasets.
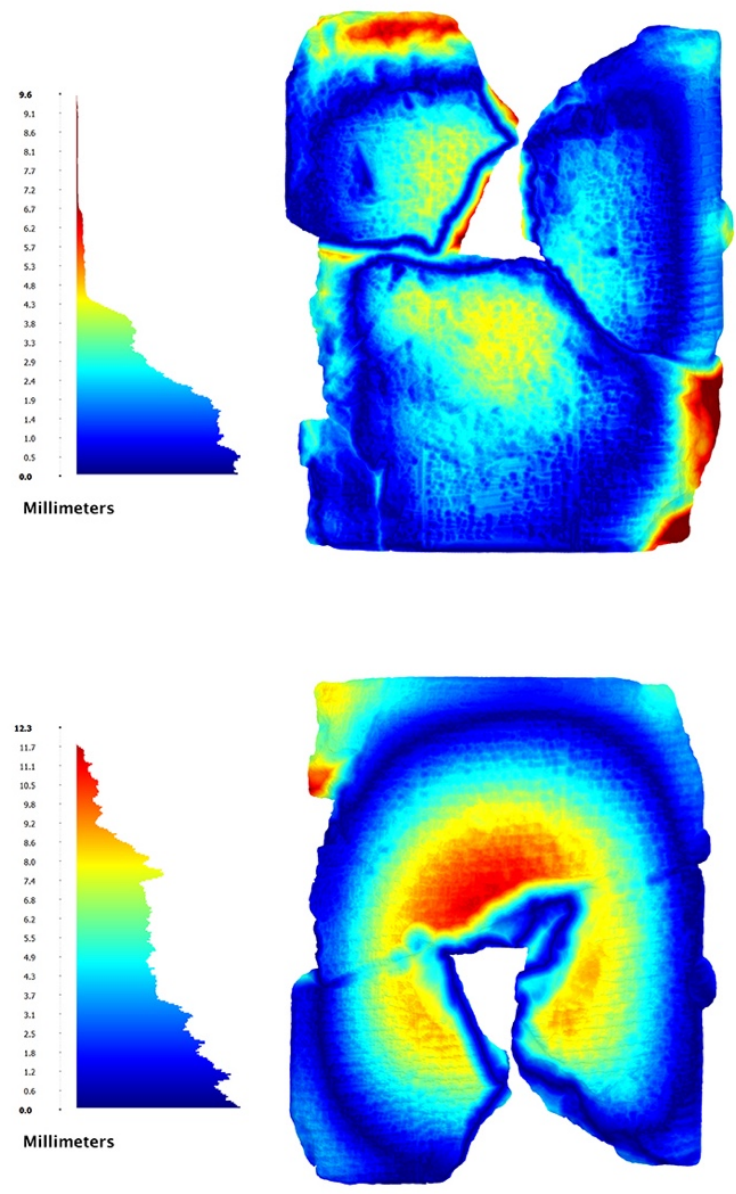

Figure 4. Color representations of Hausdorff distances from the obverse and reverse photometric stereo 3D models to the structured light 3D model.

3.1.2 Geometric Detail: One manner of visualizing geometric detail is colorization according to discrete curvature (Meyer et al., 2002). This calculation conveys variation of curvature across a surface. Comparison of the 3D models, colorized according to root mean square (RMS) (Fig. 5), Gaussian, Mean and Absolute (ABS) curvature, reveals common trends and individual disparities resultant from the different acquisition technologies. 


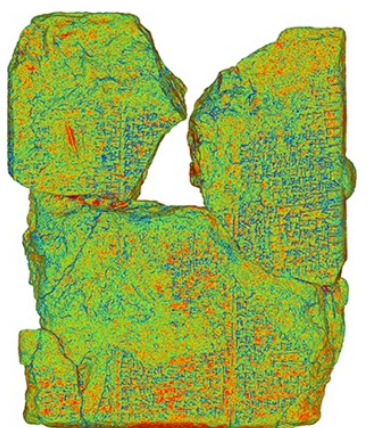

$\mathrm{a}$
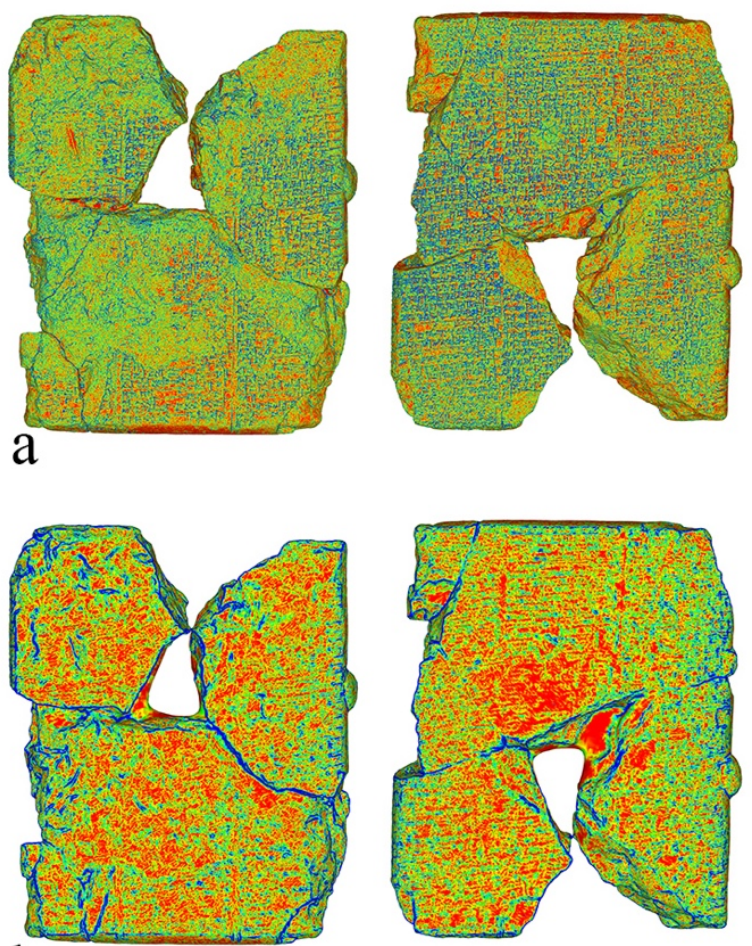

$\mathrm{b}$
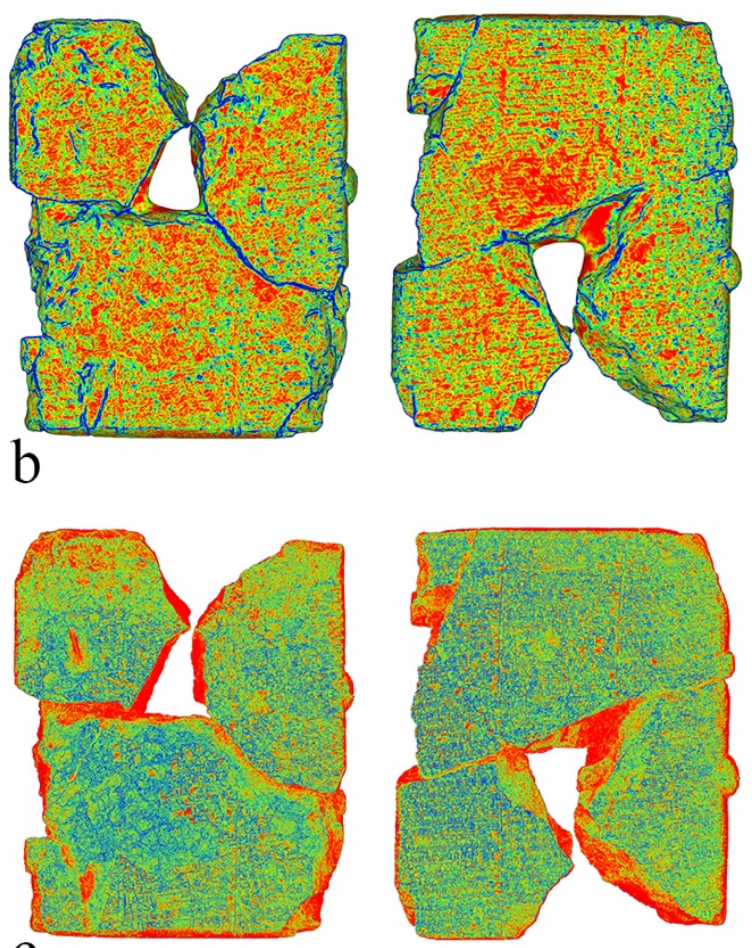

$\mathrm{C}$
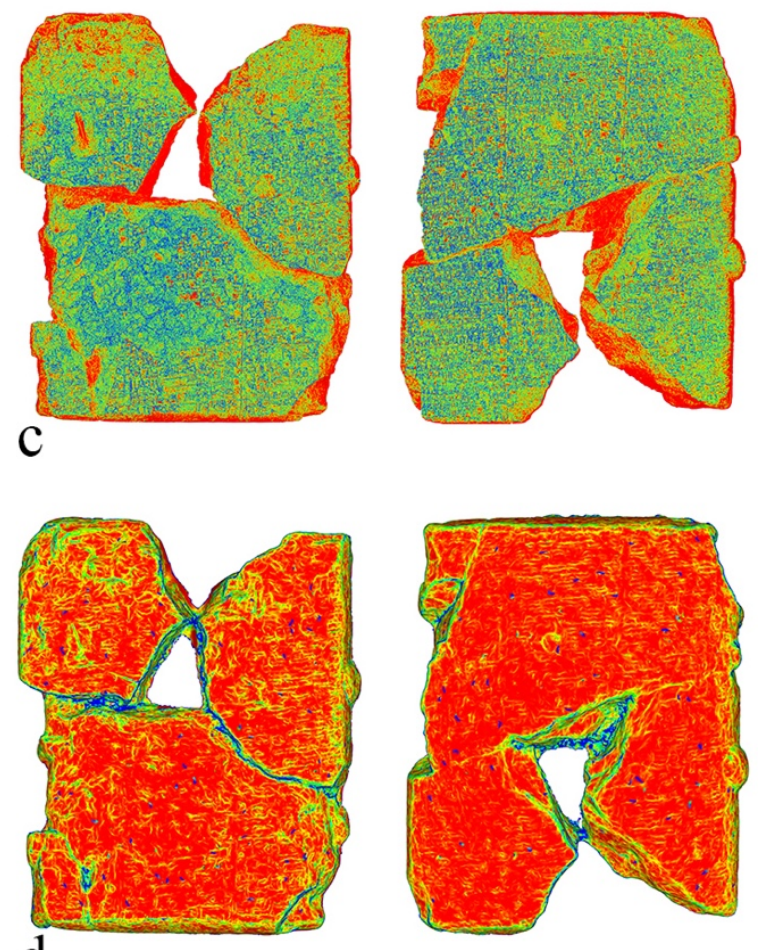

d

Figure 5. Root Mean Square discrete curvature of obverse and reverse sides of the 3D models of YBC 2178: a. structured light, b. triangulation laser scanner, c. photometric stereo, d. close-range photogrammetry.

This method of representing curvature visually allows for enhanced perception of the very detailed geometry of the 3D model created via structured light scanning (Fig. 5: a). The lack of detail in the triangulation laser scanner 3D model (Fig. 5: b) is reflected in the sparse color change in the curvature representation. Although the SG1002 is a high resolution scanhead, it is best suited for the acquisition of mid to larger shapes. Due to the tablet's size, it is on the periphery of the range for that scanner. Other, less detailed objects of a similar size have been successfully captured with the SG1002 scanhead, however, some difficulties were experienced in capturing the intricacies of the low relief inscriptions of YBC 2178. Both acquisition campaigns yielded similar results. The 3D from photometric stereo is a hybrid. As we have previously established, global distortions yield dimensional inconsistencies. This is further demonstrated by the concentration of red at the perimeters of areas with relatively drastic changes in height (Fig. 5: c). The geometry of the inscription is quite detailed locally. Finally, the representation of curvature on the CRP model conveys a lack of detail, which may be attributed to difficulties with lighting and focus.

Viewed with shaders to accentuate geometry, the range of detail in the models is starkly apparent (Fig. 6). Ambient Occlusion and Radiance Scaling were chosen to portray the fine-grained morphology of the 3D models (Vergne et al., 2010). The 3D model with the most sound geometry was yielded via structured light scanning. This 3D model is accurate in terms of dimension and detail. In contrast, the models created via triangulation laser scanning, photometric stereo and CRP had issues with the resolution of fine details and global distortion.

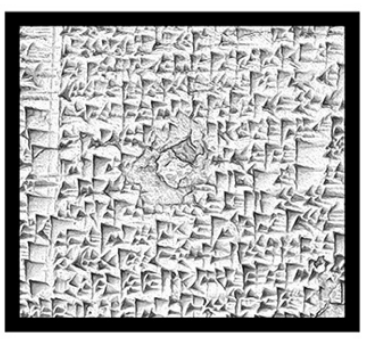

a

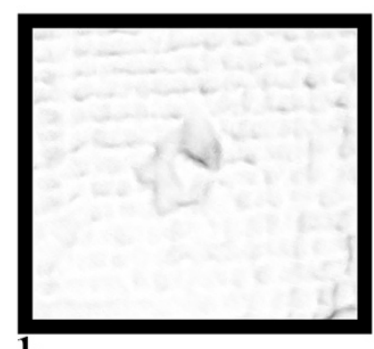

$\mathrm{b}$

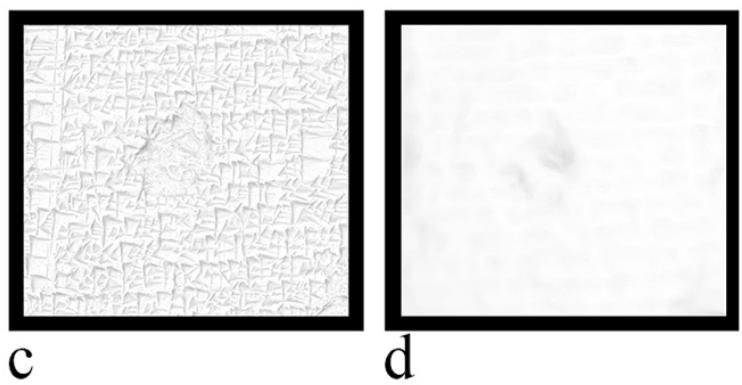

Figure 6. An area of detail from the reverse side on the 3D models of YBC 2178 visualized with Ambient Occlusion and Radiance Scaling shaders: a. structured light, b. triangulation laser scanner, c. photometric stereo, d. close-range photogrammetry.

\subsection{Texture}

Certain 3D digitization techniques capture texture, while others focus on geometric acquisition. Each acquisition technique adopted in the context of this project, excluding the triangulation laser scanner, captured texture information. High-resolution images captured with a Hasselblad H4D-200MS digital medium format camera (Fig. 1) were later texture mapped onto the model that was created from triangulation laser scanning. 
It is difficult to assess the fidelity of the textures of the 3D models in any quantitative manner. The quality of this information, can be discussed in terms of detail and resolution (Fig. 7). The color information captured with the structured light scanner is even and represents the nuances of the surface of the tablet as the clay has acquired a patina with age, handling and conservation treatment (Fig. 7: a). The scanning system was able to pick up quality data even for obstructed areas. Data texture mapped from multiple images of YBC 2178 on the triangulation laser scanning model has lost some sharpness in projection (Fig. 7: b). As these images were color corrected, the color temperature can be deemed correct, although it appears a bit more red than the others. The initial imaging campaign had its own set of objectives which did not emphasize coverage of difficult geometry. As a result, some areas in the center remain without color. The 3D models processed from RTI datasets are clad in very detailed texture (Fig. 7: c). The light array of the RTI dome used for capture contains 45 halogen lamps which illuminated the tablet consistently and resulted in clear and seemingly accurate color. These models reflect the intricacies of tablet well. Color information for the 3D model generated via CRP was not without some issues (Fig. 7: d.). The lack of sharpness and variation of color are directly tied to the resultant resolution of the model which may be attributed to the same problems with focus and lighting.

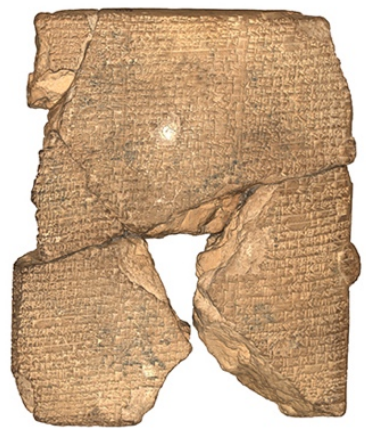

a

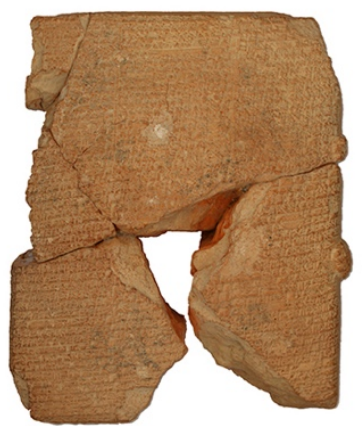

C

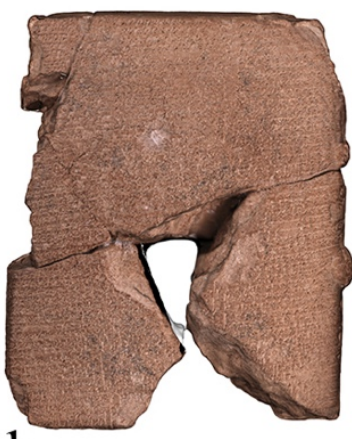

b

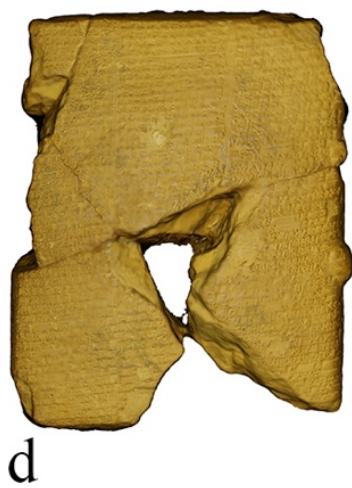

Figure 7. Textured 3D models of YBC 2178: a. structured light, b. triangulation laser scanner, c. photometric stereo, d. closerange photogrammetry.

\subsection{Dissemination}

Very high-resolution 3D models are capable of retaining much of the fidelity of an object. As such, they serve as powerful digital surrogates that enable technical study and guide conservation approaches. Most dissemination platforms, however, are not able to accommodate extremely large file sizes or exceedingly dense point clouds. Lower resolution models can be fashioned for 3D printing or web visualization, which enable engagement across the distances.

3.3.1 3D Printing: A Dremel 3D20 Idea Builder extrusion 3D printer was available for translation of the $3 \mathrm{D}$ models back into a tangible form. This printer uses $1.75 \mathrm{~mm}$ PLA filament and is capable of printing layers with a thickness of $0.10 \mathrm{~mm}$. The maximum volume of a build is $230 \times 150 \times 140 \mathrm{~mm}$. 3D prints scaled at $50 \%$ and set for the highest quality with an infill of $25 \%$ were carried out for the models created from both scanning techniques. Both prints were of created from around 700 layers and took an estimated 6 hours and 24 meters of material. These models contrast greatly in quality. Due to the scale of the prints, this difference in 3D model quality is reflected generally in smoothness of the 3D print and not discernible detail. The 3D models created via photometric stereo required further processing and an addition of a base to present a model thick enough for print.

3.3.2 Web-hosted Visualization: The outputs from the digitization methods chosen for this project had file sizes that exceed standard requirements for incorporation in web-hosted viewers. Downsampled versions were created and uploaded onto Sketchfab. Sampling, remeshing and attribute transfer pipelines in MeshLab enabled downsampled models to best maintain the integrity of the original builds while also allowing for engagement across the distances.

\section{DISCUSSION}

3D models have become popular for their ability to bring cultural heritage closer to and more tangible to researchers and the public alike. The applications of 3D models run the gamut from virtual technical analyses to classroom engagement. Due to the variety of possibilities for interaction that $3 \mathrm{D}$ data presents, there are many considerations to be taken into account when capturing and building 3D models.

This paper tries to provide answers as to the efficiency and efficacy of different acquisition techniques to create final 3D models for different applications in research and dissemination. It also delves into the restrictions in data capture for production imposed by these methods. A case study of 3D model creation for YBC 2178 was done by different techniques and at different times. Triangulation laser scanning and RTI were carried out in the IPCH Digitization Laboratory under carefully controlled environmental conditions (lighting, humidity, heat etc.) and without strict limitations on time. CRP and structured light scanning were done on site at the Yale Babylonian Collection, which underlined the importance of the planning for the time and optimization of present conditions such as ambient lighting.

The differences between geometric and textural details play an important role in the decision of the use of the $3 \mathrm{D}$ model. For instance, a one to one scaled, colored 3D print of YBC 2178 could be used for a better holistic understanding of the object itself. This kind of a print would be very helpful for the cuneiform experts who might read the text and appreciate a physical representation of the tablet. For such a print both the geometry and the texture would be equally important. The 3D model generated via structured light would be a good selection for $3 \mathrm{D}$ printing since it has the highest geometric fidelity and quite consistent, accurate texture. For a smaller scaled, colorless $3 \mathrm{D}$ prints that could be incorporated into an exhibition or lesson 
plan as a tactile handout, the geometry yielded via triangulation laser scanner would suffice.

Dissemination via the web would be served by all means of digitization presented by this study. Downsampled versions of the models could be incorporated on different web-hosted viewers so long as they are accompanied by information related to their creation and resultant accuracy. Models yielded via photometric stereo should be linked to clear information regarding their limitations.

Although these photometric stereo models present very fine details, the distortion in the models make them unreliable in terms of geometric precision. Since this data can be generated from an RTI dataset and easily processed, it can be generated for little investment. This data has the potential to aid epigraphers who are most concerned with more localized details of inscriptions and would benefit greatly from the capability of applying geometry enhancing filters and lighting angles in 3D visualization software and on the web. In this case, the epigraphers would not be bereft of the possibility to make meaningful measurements.

The ability to derive precise measurement from 3D data is especially appealing to conservators, who wish to monitor small scale changes in materials over time. For such work, a repeatable pipeline that yields an extremely high fidelity, high resolution geometry without being too resource intensive is necessary. In the context of this study, structured light scanning stands out as the best option for creating a benchmark of an object and at specific points in time for monitoring.

CRP is a cost-efficient method to be used when there is a limited time for data capture. However, a non-uniform background in the environment and uncontrollable lighting conditions resulted in long, tedious hours of pre-processing while still the finest details were not detectable (Fig. 6). Due to the fact that this method of digitization can be easily influenced by environmental conditions, it might yield variable results and therefore is not perhaps reliably repeatable without standardized protocols and measurable controls. Strives toward such protocols are continually being made (Mudge and Schroer, 2017).

\section{REFERENCES}

ASP (American Society of Photogrammetry), 1966. Manual of Photogrammetry, 3rd Edition, Vol. I and II.

Besl, P. J., McKay, N. D., 1992. Method for registration of 3-D shapes. In: IEEE Transactions on Pattern Analysis and Machine Intelligence, Vol. 14, Issue 2, pp. 239-256.

Cignoni, P., Rochinni, C., Scopigno, R., 1998. Metro: measuring error on simplified surfaces. In: Computer Graphics Forum, Vol. 17, No. 2, pp.167-174.

Cignoni, P., Callieri, M., Corsini, M., Dellepiane, M., Ganovelli, F., Ranzuglia, G., 2008. MeshLab: an open-source mesh processing tool. In: Proceedings of the Sixth Eurographics Italian Chapter Conference, Salerno, Italy, pp. 129-136.

Corsini, M., Cignoni, P., Scopigno, R., 2012. Efficient and flexible sampling with blue noise properties of triangular meshes. In: IEEE Transactions on Visualization and Computer Graphics, Vol. 18, Issue 6, pp. 914-924.
Fassi, F., Fregonese, L., Ackerman, S., De Troia, V., 2013. Comparison between laser scanning and automated 3D modeling techniques to reconstruct complex and extensive cultural heritage areas. In: The International Archives of the Photogrammetry, Remote Sensing and Spatial Information Sciences, Trento, Italy, Vol. XL-5/W1, pp. 73-80.

Kazhdan, M., Hoppe, H., 2013. Screened poisson surface reconstruction. In: ACM Transactions on Graphics, Vol. 32, Issue 3, Article 29.

Luhmann T., Robson, S., Kyle, S., Harley E., 2006. Close Range Photogrammetry, Principles, techniques and applications. Whittles Publishing.

Matthews, N. A., Noble, T. A., Breithaupt B. H., 2004. From dinosaur tracks to dam faces: a new method for collecting threedimensional data. In: Geological Society of America Denver Annual Meeting.

Menna, F., Nocerino, E., Remondino, F., Dellepiane, M., Callieri, M., Scopigno, R., 2016. 3D digitization of an heritage masterpiece - a critical analysis on quality assessment. In: The International Archives of the Photogrammetry, Remote Sensing and Spatial Information Sciences, Prague, Czech Republic, Vol. XLI-B5, pp. 675-683.

Meyer, M., Desbrun, M., Schröder, P., Barr, A. H., 2002. Discrete differential-geometry operators for triangulated 2manifolds. In: Visualization and Mathematics III, pp. 35-57.

Mudge, M., Malzbender, T., Schroer, C., Marlin, L., 2006. New reflection transformation imaging methods for rock art and multiple-viewpoint display. In: The 7th International Symposium on Virtual Reality, Archaeology and Intelligent Cultural Heritage, pp. 195-202.

Mudge, M., Schroer, C., 2017. Challenges and insights from the photogrammetric capture of a large-scale Diego Rivera fresco. Presented at: The $45^{\text {th }}$ Annual Computational Applications and Quantitative Methods in Archaeology Conference, Atlanta, GA, United States of America, 15 March.

Pintus, R., Podda, S., Vanzi, M., 2006. An automatic alignment procedure for a 4-Source photometric stereo technique applied to scanning electron microscopy. In: Proceedings of the TwentyThird IEEE Instrumentation and Measurement Technology Conference, Sorento, Italy, pp. 1278-1283.

Ranzuglia, G., Callieri, M., Dellepiane, M., Cignoni, P., Scopigno, R., 2013. MeshLab as a complete tool for the intergration of photos with high resolution $3 \mathrm{D}$ geometry data. In: Proceedings of the $40^{\text {th }}$ Conference in Computer Applications and Quantitative Methods in Archaeology, Southampton, United Kingdom, pp. 406-416.

Vergne, R., Pacanowski, R., Barla, P., Granier, X., Schlick, C., 2010. Radiance scaling for versatile surface enhancement. In: Proceedings of the 2010 ACM SIGGRAPH Symposium on Interactive 3D Graphics and Games, Washington, DC, United States of America, pp. 143-150.

Zhang, Z., 1994. Iterative point matching for registration of freeform curves and surfaces. In: International journal of Computer Vision, Vol. 13, Issue 2, pp. 119-152. 